\title{
Qualidade de Vida e Ambient Assisted Living: modelos de inclusão para adultos com deficiência
}

\author{
Quality of Life and Ambient Assisted Living: \\ inclusion models for adults with disabilities
}

\author{
CATIA GIACONI \\ Università degli Studi di Macerata \\ MICHELA CARBONARI \\ Università degli Studi di Macerata
}

\begin{abstract}
Resumo: O passar do tempo impõe vários desafios com relação aos cuidados necessários aos adultos com deficiência. Pensemos nas diversas fases de transição ao longo da vida, que podem marcar o futuro da pessoa com deficiência: a finalização do período escolar, a inserção em novos ambientes sociais e de trabalho, mudança de moradia, ou, a própria permanência no contexto familiar após a escola. Seja qual for a possibilidade, nem sempre é consonante com as necessidades das pessoas envolvidas. Para que essas mudanças possam ser melhor vivenciadas pela pessoa com deficiência e por sua família, podemos contar com pesquisas e experimentos com tecnologias assistivas. Essas experiências têm possibilitado autonomia e a convivência em diferentes ambientes sociais. Essas práticas não enfatizam somente a aplicação de tecnologia, mas, acima de tudo, a reflexão e busca contínuas de Qualidade de Vida (QV). Esse artigo traz reflexões sobre percursos e ações, em projetos italianos e europeus que buscam associar tecnologias com QV, onde as tecnologias e ambientes de vida assistivos têm papel central, como modelos de inclusão, independência, participação e bem estar.
\end{abstract}

Palavras-chave: Qualidade de vida. Fases evolutivas. Pessoa com deficiência. Tecnologias Assistivas. Ambient Assisted Living

Abstract: Advancing age impose several challenges to the necessary care to adults with disabilities. There are various transition phases throughout life, they can mark the future of the disabled person: the completion of schooling, then try in to new social and work environments, change of residence, or the permanence within the family after school. Whatever the variable, it may notal ways bein line with the need soft he people involved. So that these changes can be best experienced by the disabled person and his/her family, as seen in some research and experiments with assisted technologies. These experiences have allowed autonomy and living in different social environments. These practices not only emphasize the application of technology but, above all, the continuous reflection and search of Quality of Life (QoL). This article reflects on path ways and actions, in Italian and European projects that seek to associate technology with QoL, where technologies and assisted living environments play a central role, as inclusion models, possibilities of independence, participation and well being.

Keywords: Quality of life. Development phases. Disable person. Assisted Technologies. Ambient Assisted Living.

GIACONI, Catia; CARBONARI, Michela. Qualidade de Vida e Ambient Assisted Living: modelos de inclusão para adultos com deficiência. Informática na Educação: teoria \& prática, Porto Alegre, v. 19, n. 2, p. 50-70, jun./set. 2016. 


\section{Introdução: o avançar da idade, entre desafios e emergências pedagógicas}

O avanço da idade das pessoas com deficiência impõe notáveis desafios, sobretudo se considerarmos alguns fatores emergentes e de particular interesse, como o cuidado de adultos com deficiência ao longo da vida por meio de soluções e tecnologias assistivas, que permitem uma maior autonomia e possibilidades de convivência em vários ambientes sociais. Esse quadro apresenta diferentes níveis de complexidade e, portanto, pode ser interpretado por diversas chaves de leitura.

Alguns autores levam em consideração as consequências sociais, portanto, questões sobre bem estar individual e custos econômicos e sociais (THOMPSON et al., 2012). Nesse caso, a abordagem interdisciplinar visando a implementação de ambientes assistivos de vida tende a demonstrar como essas soluções podem, com o tempo, abater os custos dos serviços sociais e sanitários, que são afetados pelo aumento das populações idosa e com deficiência e devem gerir recursos sempre mais escassos.

Na perspectiva da pedagogia especial somam-se significativos desafios educativos. Faz-se necessário refletir sobre percursos e ações, visando projetos que levem em conta a Qualidade de Vida (QV), onde as tecnologias e ambientes de vida assistivos têm papel central, como modelos de inclusão, independência, participação e bem estar (GIACONI; RODRIGUES, 2014).

Nessa área de estudo, muitas são as questões centrais colocadas em evidência pela literatura científica e concentram-se, de modo particular, na delicada construção de projetos e soluções personalizadas relacionadas com a QV de pessoas adultas com deficiência (GIACONI; RODRIGUES, 2014; PENNE et al., 2012; MAES et al., 2007; ZIJLSTRA; WASKAMP, 2005; CAMBRIDGE; CORNABY, 2000; FELCE et al., 2000).

As emergências pedagógicas implicam na individuação das fases críticas do desenvolvimento de pessoas com deficiência e, que ainda hoje, correm o risco de serem resolvidas como soluções emergenciais, mais do que por meio de percursos projetados desde a infância.

Pensemos nas diversas fases de transição que podem marcar, de um modo ou de outro, o futuro da pessoa com deficiência. Ela e sua família encontram alguns fatores críticos, como a finalização do período escolar e a inserção em vários ambientes sociais e de trabalho, ou, na ausência dessa possibilidade, a mera permanência no contexto familiar. Posteriormente, existe a possibilidade de mudança de moradia, ou seja, a passagem do contexto familiar para outras realidades e situações de habitação, nem sempre consonantes com suas necessidades.

Ao término do período escolar, para a pessoa com deficiência existe o risco de permanecer exclusivamente no ambiente familiar que, com o passar do tempo, principalmente devido ao progressivo envelhecimento dos pais, pode não fornecer os apoios necessários para uma vida de qualidade. A isso, com frequência, associa-se uma outra passagem delicada de perda da rede de pessoas da escola e da família, por condições delicadas de saúde ou pela morte dos pais e parentes próximos. 
Mesmo nesses casos, a fase de transição da própria casa para a de outros familiares (irmãos) ou, com frequência, para estruturas residenciais especializadas, é uma passagem complexa, nem sempre suficientemente preparada do ponto de vista da QV das pessoas com deficiência. Com o tempo, o sujeito com deficiência corre o risco de ter menos ocasiões de integração e de participação na comunidade. Sua socialização, comumente, já é restrita a sua casa e a um ambiente social circunscrito e, se vai viver em clínicas especializadas, corre maior risco de se relacionar somente com pessoas com deficiência e de ter seu espaço físico de ação restrito à estrutura do local.

Numerosos estudos evidenciam que os adultos com deficiência passam a maior parte do tempo livre em casa, ou em institutos de grandes dimensões e que permanecem sós ou com familiares (GIACONI, 2015). Essas constatações apontam para o perigo de deterioração de capacidades anteriormente adquiridas.

É evidente o fato de que uma pessoa adulta com deficiência, mais do que pelo processo natural de envelhecimento, pode incorrer em deterioração precoce de suas funções cognitivas devido à passagem de um período escolar de intensa inclusão, estimulação e reabilitação, para um outro onde passa a ter escasso contato com pessoas da mesma idade, limitado repertório de atividades recreativas e de lazer, assim como falta de inclusão laboral e reabilitação adequada. Essas mudanças intervêm de modo relevante sobre a QV e, em específico, sobre a experiência de vida decorrente. Dessa forma, justifica-se a contínua ênfase em projetos e modelos de inclusão, que garantam níveis adequados de QV e de participação social.

Mesmo conscientes da complexidade da questão, propomos analisar nesse artigo três aspectos cruciais da inclusão de adultos com deficiência:

a) o modelo da Qualidade de Vida, para analisar as necessidades de adultos com deficiência;

b) o Ambient Assisted Living, como modelo para a inclusão;

c) projetos e personalização de trajetórias de vida de pessoas com deficiência, com o apoio de tecnologias assistivas.

\section{A Qualidade de Vida Como Modelo de Análise}

Os numerosos estudos focados na QV, presentes na literatura científica, trazem uma pluralidade de definições e numerosos procedimentos capazes de medir a Qualidade de Vida (SCHALOCK; VERDUGO ALONSO, 2002; SCHALOCK, 2000; FELCE; PERRY, 1996). A variedade de conceituações reflete, por um lado, as diferentes abordagens disciplinares (filosófica, econômica, médica, social, pedagógica, psicológica, etc.) e, por outro, as diversas orientações epistemológicas que valorizam as condições objetivas ou, ao contrário, o levantamento de indicadores subjetivos.

Entre essas abordagens teóricas, alguns autores polarizam a atenção majoritariamente sobre aspectos objetivamente mensuráveis ${ }^{1}$, como por exemplo, as condições de vida com

\footnotetext{
1 Incluem-se, sobretudo, os estudos em âmbito médico e sociológico, que polarizam a atenção sobre variáveis da QV como um estado de saúde objetivamente detectável, a renda individual, a qualidade e a quantidade de cuidados médicos recebidos.
} 
relação à saúde física, o ambiente, as relações sociais, a renda e a atividade laboral (GIACONI, 2015; LANDESMAN, 1986); outros levam em consideração os aspectos subjetivos ${ }^{2}$ e de satisfação pelas próprias condições, o bem estar psicológico, as aspirações, os valores e as expectativas da pessoa (BROWN, I.; BROWN, 2009; BORTHWICK-DUFFY, 1992).

Pesquisas recentes conduzem à consideração da QV como um construto complexo constituído de uma pluralidade de áreas relevantes, sejam de caráter objetivo que subjetivo. Principalmente no fim dos anos noventa foram propostas perspectivas de síntese entre os dois âmbitos anteriores da QV. Tal posição, que considera ambas dimensões da vida de adultos com deficiência, é representada pelos primeiros estudos de Brown (1989). Como componentes objetivos, o autor evidencia os aspectos que poderiam garantir altos níveis de QV:

- Presença de adequadas características do ambiente físico;

- Atenção às condições alimentares e de saúde da pessoa;

- Quantidade e qualidade de treinamento e de apoio reais, presentes na estrutura (BROWN, 1989, p. 66).

Como dimensão subjetiva, o autor leva em consideração:

- A percepção da pessoa quanto aos apoios, ao próprio estado de saúde, o envolvimento na comunidade, etc.;

- As expectativas;

- O nível de satisfação das pessoas com deficiência (BROWN, 1989, p. 82).

Trata-se de uma conceituação que marca um significativo avanço no paradigma da QV. A área, certamente se ressente da presença ou não de alguns requisitos objetivos, mas, ao mesmo tempo, é estreitamente conectada às expectativas e ao nível de satisfação que a pessoas manifestam frente as suas atuais condições de vida. $\mathrm{O}$ aspecto inovador da proposta de Brown (1989), pensamos, consiste em considerar a QV como determinada tanto pelo lacuna entre desejos e necessidades satisfeitas ou não, quanto pelo controle que a pessoa consegue exercitar sobre o próprio ambiente.

Para analisar a QV como fenômeno multidimensional, Schalock e colaboradores propõem os seguintes conceitos chave: domínios, indicadores e descritores (SCHALOCK et al., 2002, SCHALOCK; VERDUGO ALONSO, 2002). Os domínios de base seriam âmbitos ou dimensões relevantes na vida de uma pessoa. Alguns domínios da QV seriam: o bem estar emocional, as relações interpessoais, o bem estar material, o desenvolvimento pessoal, o bem estar físico, a autodeterminação, a inclusão social e os direitos (SCHALOCK; GARDNER; BRADLEY, 2007).

Esses domínios são aspectos fundamentais para definir QV e, de fato, se faltassem na vida de uma pessoa inviabilizariam, ou dificultariam muito, as possibilidades de desenvolvimento pessoal. Mesmo na vida de adultos com deficiência, a ausência de uma rede eficaz de relações e a falta de participação na vida comunitária condicionariam a possibilidade de satisfazer as condições de vida.

Para sair de uma mera definição abstrata e, portanto, para operacionalizar os domínios da QV os autores estabelecem indicadores. Os indicadores caracterizam as dimensões de QV, ou seja, as condições específicas a serem consideradas em cada domínio (SCHALOCK; VERDUGO

\footnotetext{
${ }^{2}$ Essa perspectiva è particularmente desenvolvida em estudo de âmbito psicológico. Os aspectos de QV considerados estão estreitamente relacionados à percepção que a pessoa tem das próprias condições de vida.
} 
ALONSO, 2002, p. 67). Em outras palavras, como é possível qualificar os domínios da QV, por exemplo, os domínios de bem estar físico e da autodeterminação?

No primeiro caso, nos referimos à avaliação da presença de uma adequada condição geral de saúde, de correta alimentação, higiene pessoal e significativas atividades recreativas. No segundo, se tenta avaliar o nível de participação aos processos decisórios que se referem a aspectos cotidianos e gerais de sua existência. Esses são indicadores que caracterizam os domínios do bem estar físico e da autodeterminação, que permitem concretamente especificar o significado geral das duas dimensões da QV.

É importante ressaltar que os indicadores dos domínios podem variar individualmente em termos valorativos, de importância e de percepção. Por exemplo, uma pessoa poderia ter um objetivo e um estado de saúde dado, mas ter uma sensação geral de mal estar físico. Ou ainda, nos diversos domínios das relações interpessoais um sujeito pode, ao longo de sua vida, considerar mais importante, ou atribuir um valor maior, às relações com os amigos, mais do que aos cuidados médicos e às relações familiares.

Com referência a recentes meta-análises, Schalock e Verdugo Alonso (2006) apresentam uma revisão sistemática dos domínios e dos indicadores, que emergem da análise das diversas teorias do paradigma da QV. O quadro $1^{3}$ traz exemplos de domínios e de indicadores da QV. Certamente os indicadores não são suficientes para revelar as especificidades das pessoas e dos ambientes da vida cotidiana. Os indicadores podem ser declinados nos diversos contextos e nas específicas situações, por meio da individualização de descritores, ou seja, de comportamentos ou situações observáveis e relevantes para o contexto em questão.

Quadro 1 - Exemplo de Domínios e de Indicadores.

\begin{tabular}{|l|l|}
\hline Domínios & Indicadores \\
\hline I nclusão social & $\begin{array}{l}\text { Integração na comunidade, participação } \\
\text { na comunidade, papel, apoios, } \\
\text { aceitação. }\end{array}$ \\
\hline Bem estar físico & $\begin{array}{l}\text { Alimentação, higiene pessoal, saúde; } \\
\text { assistência de saúde; tempo livre. }\end{array}$ \\
\hline Relações interpessoais & $\begin{array}{l}\text { Relações familiares, relações de } \\
\text { amizade, relações de trabalho e sociais, } \\
\text { intimidade. }\end{array}$ \\
\hline Bem estar material & $\begin{array}{l}\text { Bens, propriedades, ocupação, situação } \\
\text { financeira, acomodação, segurança. }\end{array}$ \\
\hline Bem estar emocional & $\begin{array}{l}\text { Satisfação, autoconceito, segurança, } \\
\text { espiritualidade, felicidade. }\end{array}$ \\
\hline Auto- determinas I one & $\begin{array}{l}\text { Opiniões e preferências pessoais; } \\
\text { decisões e escolhas; controle pessoal. }\end{array}$ \\
\hline Desenvolvimento pessoal & $\begin{array}{l}\text { Enriquecimento pessoal, habilidades, } \\
\text { competências pessoais, realização. }\end{array}$ \\
\hline Direitos & $\begin{array}{l}\text { Conhecimento sobre direitos, tutela, } \\
\text { privacidade, confidencialidade, respeito. }\end{array}$ \\
\hline
\end{tabular}

Fonte: Adaptação de Schalock; Verdugo Alonso (2006).

\footnotetext{
3 Os critérios para a seleção dos indicadores devem ser (SCHALOCK, 2006): validade (devem medir o que queremos medir); confiabilidade (acordo entre sujeitos e avaliadores diversos); sensibilidade (mede as mudanças); especificidade (reflete as situações); sustentabilidade (realismo em termos econômicos e de recursos humanos disponíveis, em uma ótica temporal extensa); avaliável longitudinalmente (coerência entre os indicadores); sensibilidade cultural (reflete a cultura de pertencimento).
} 
Podemos pegar como exemplo o domínio da autodeterminação, no qual encontramos o indicador da escolha e decisão. Nesse caso, podemos individuar como descritor a presença de opções de escolha entre as atividades cotidianas e de tempo livre, entre os alimentos nas refeições, entre as roupas para vestir, entre os lugares para visitar, entre as pessoas para frequentar, etc. Essas situações seriam observadas nos contextos de vida, familiares ou residenciais da pessoa com deficiência, como características relevantes para o indicador de decisão e escolha, ou seja, da dimensão de autodeterminação da pessoa com deficiência.

Com a individuação dos descritores completa-se o processo de operacionalização dos domínios da QV. O exercício de identificação dos domínios, dos relativos indicadores e descritores levaria diretamente à avaliação dos resultados pessoais, dos programas e dos serviços às pessoas com deficiência, em termos de QV (BROWN, I.; BROWN, R. I., 2009; SCHALOCK; GARDNER; BRADLEY, 2007; EMERSON et al., 2004).

De acordo com essas fases, de identificação e de operacionalização dos domínios, são construídos os diversos instrumentos de pesquisa como questionários, índices, entrevistas, etc. Como veremos mais adiante, esses procedimentos são focados na análise objetiva das condições de vida, assim como no estudo da satisfação da pessoa com relação à QV.

Algumas perspectivas individuam áreas transversais nas quais inserir e considerar as diversas dimensões da QV. Entre essas consideramos interessante colocar em evidência a proposta de Renwick e Brown (1996), até porque na Europa o projeto da Universidade de Toronto é adotado com frequência, mesmo em associações e organizações que se ocupam, por exemplo, de adultos com espectro autista.

Na teorização, os autores definem a QV como o grau de satisfação por meio do qual as pessoas usufruem de possibilidades e de oportunidades consideradas importantes para as suas existências (RENWICK; BROWN, 1996). Nessa definição os autores desenvolvem o construto de QV em três grandes áreas transversais: ser (being), pertencer (belonging) e tornar-se (becoming). Essas áreas indicam as características individuais e específicas de uma pessoa (ser), a adaptação e a qualidade de inclusão nos contextos de pertencimento (pertencer), a projeção e o desenvolvimento das pessoas no curso da vida (tornar-se).

A macro área, sobre a qual concentraremos nossa atenção, é explicitada em níveis prático, recreativo e evolutivo. Para o âmbito prático os principais indicadores de referência são as atividades doméstica, escolares e laborais; para o recreativo são consideradas relevantes as atividades e recursos para o tempo livre; para a área evolutiva são considerados a aprendizagem contínua e a capacidade de adquirir habilidades para melhorar as relações e adaptar-se a ambientes e situações diversas.

Nessa mesma direção, propomos a perspectiva 3C de Cottini e Fedeli (2007). Os autores propõem três aspectos transversais para a análise das dimensões da QV: centralidade, controle e continuidade. A centralidade permite avaliar se a estrutura e as intervenções educativas estão orientadas a partir das características fundamentais da QV das pessoas com deficiência. As áreas transversais de controle e continuidade permitem avaliar se esses aspectos são potencialmente controláveis pela pessoa ou se estão presentes em termos de tempo e continuidade em seu projeto de vida. 
Schalock e outros (2010) implementaram a sua proposta chegando a inserir oito dimensões de QV, em três áreas transversais: independência, participação social e bem estar. A independência refere-se ao domínio do desenvolvimento pessoal e da autodeterminação; a participação social, os domínios das relações interpessoais, inclusão social e direitos; o bem estar compreende bem estar físico, material e emotivo.

Consideramos que a abordagem desses estudos dirigidos à individuação de áreas transversais, seja particularmente significativa para analisar a QV de pessoas com deficiência e para a avaliação de projetos educativos visando manter e melhorar os níveis de QV. Essas investigações permitem individuar as áreas relevantes para intervenções educativas, assim como indicadores gerais para projetar e monitorar as características centrais da QV (como condições de habitação, inclusão laboral, etc.). As linhas-guia internacionais consideram essas orientações aplicáveis a todas as pessoas, incluindo as com deficiência (BROWN, I.; BROWN, R. I., 2009; REITER; SCHALOCK, 2008; SCHALOCK et al., 2002).

Foi demonstrado que as características de QV próprias de outros grupos da população são compartilhadas pelas pessoas com deficiência (EMERSON et al., 2004; DENNIS et al., 1993). Porém, é importante ter presente que existem alguns aspectos estritamente ligados à QV de pessoas com deficiência, referentes à saúde e ao desenvolvimento cognitivo (BORTHWICKDUFFY, 1992; FLANAGAN, 1978), o tipo de deficiência, com eventuais quadros de deficiência múltipla e a idade (BROWN, R. I., 1988).

Os domínios fundamentais são os mesmos para todas as pessoas, mas o conteúdo pode variar individualmente, em termos de valores e importância. É indiscutível, portanto, a posição que demonstra como esses domínios são avaliados diversamente pelos indivíduos e como o valor a esses atribuídos mudem ao longo da vida (SCHALOCK; VERDUGO ALONSO, 2006; CAMPO et al., 1996), assim como é compartilhada a relevância do papel das experiências, dos diversos sistemas e contextos que influenciam nossas vidas (SCHALOCK et al., 2011, PETRY; MAES; VIASKAMP, 2009, SCHALOCK; VERDUGO ALONSO, 2002). Além disso, a QV não se refere a um momento fixo de tempo, mas cobre o inteiro percurso de vida (BROWN, R. I., 1997).

A QV é sensível a uma dimensão temporal, uma vez que a realização e percepção da mesma mudam potencialmente sobre um eixo diacrônico, nos diversos períodos de vida e, em um eixo sincrônico, nos diversos espaços de existência da pessoa.

Consideramos importante para qualquer estudioso e profissional o exercício de individualização dos domínios da QV, de indicadores e de descritores. Esse exercício torna-se fundamental para não utilizar de modo prescritivo as diversas propostas analisadas, mas para a aplicação do construto da QV sempre referido às pessoas e aos seus contextos.

A partir dessa modalidade operativa, é possível proceder com a construção de instrumentos de observação e de investigação (questionários, roteiros de observação, etc.) e com projetos e realização de práticas educativas direcionadas à promoção da QV de pessoas com deficiência e o planejamento de ambientes de vida que correspondam as suas necessidades e direitos de participação e inclusão social. 


\section{Ambient Assisted Living como Modelo de Inclusão}

Para trabalhar com o paradigma da QV é necessário acompanhar o percurso de vida da pessoa com deficiência, em termos de moradia e da rede integrada na comunidade a qual pertence. Para tanto, três aspectos devem ser levados em conta, para evitar simplificações nesse processo. Em primeiro lugar, é necessário recordar que a estruturação de possibilidades potenciais funda-se na nas exigências específicas da pessoa com deficiência, seu contexto e projeto de vida. Em segundo lugar, ocorre construir pontes significativas de preparação para uma vida autônoma ou em estruturas residenciais, evitando comparações com a vida em família, para que a interpretação dessas alternativas seja de uma melhora na QV.

Enfim, recordamos que, apesar de serem indicada sem pesquisas expostas nesse artigo, existem diferenças de QV entre sujeitos que vivem com suas família e outros que vivem em instituições, podendo ser pequenas e grandes estruturas (LIFSHITZ; MERRICK; MORAD, 2008). A QV também depende da capacidade de conexão dos serviços em redes significativas com as famílias, assim como depende da profissionalidade das pessoas que dirigem e operam essas estruturas (GIACONI, 2012a).

$\mathrm{Na}$ rede de apoio às famílias e às pessoas com deficiência, encontramos centros socioeducativos de reabilitação diurnos e estruturas de residência, que oferecem serviços de breve duração ou prolongados. Novas perspectivas estão sendo orientadas pelo Ambient Assited Living, ou Ambientes de Vida Assistivos, que estão configurando um modelo eficiente e eficaz de inclusão de adultos com deficiência. São formas adequadas e sustentáveis que permitem a pessoas com deficiência e a idosos de viverem em casas com segurança, apoio nas atividades cotidianas e com, consequente, autonomia e participação ativa (CASAZZA et al., 2002).

O Ambient Assisted Livingé um programa promovido pela Comissão Europeia (2007), orientado à QV de pessoas vulneráveis, por meio do apoio de tecnologias da informação e da comunicação (TIC). É baseado em uma abordagem interdisciplinar, por meio da qual são experimentadas soluções software e hardware dedicadas, dos pontos de vista ambiental e pessoal, a melhorar a QV de pessoas com deficiências e idosos.

Por exemplo, existem microssistemas portáteis, mimetizados como vestuário (cinto, relógio, sapatos, etc.), capazes de medir a dinâmica do movimento humano e monitorar a atividade física, postura, deambulação, sono e eventos como quedas. Em outras palavras, são sensores que permitem levantar a qualquer hora do dia e da noite, eventuais alterações fisiológicas e possíveis transformações que geram emergências no ambiente de vida.

Existem muitos tipos de sensores, dos gerais ambientais aos áudios e vídeos, que fornecem informações sobre atividades cotidianas das pessoas e suas condutas, podendo enviar indicações de alerta à família ou aos cuidadores. Sensores ambientais também podem ser projetados para monitoramento de eletricidade, gás, abertura e fechamento de portas e janelas, etc. A fronteira da pesquisa está sempre mais ampla, incluindo comunidades virtuais de ajuda mútua (RASHIDI; MIHAILIDIS, 2013), ou cuidador à distância para intervenções necessárias. 
Em geral, a complexidade do Ambient Assisted Living é direcionada à activity recognitione à análise comportamental, com particular ênfase aos sensores vestíveis ou aos dispositivos de vídeo, para levantamento de parâmetros fisiológicos. Os sensores ambientais, por sua vez, são mais voltados à segurança, à deambulação e mobilidade no ambiente, o acesso e uso de poltronas e camas, a solicitação de intervenção, a memorização de uma pluralidade de dados e análise de informações provenientes dos sensores, para monitorar atividades e comportamentos.

Com tudo isso, o escopo do Ambient Assisted Livingé o de prolongar a permanência da pessoa no próprio ambiente de vida, potencializando possibilidades de autossuficiência, segurança, mobilidade, autonomia e interação significativa. Essas dimensões estão em linha com a nova perspectiva europeia (HORIZON, 2020). Muitos são os programas de pesquisa nacionais e internacionais de apoio a pessoas vulneráveis, para garantir a QV e, ao mesmo tempo, reduzir os custos de assistência domiciliar ou de outro tipo necessário. Em populações conhecidas pelo seu envelhecimento, essas iniciativas são bem-vindas.

É importante repensar um sistema integrado para o cuidado do adulto com deficiência, de sua família, e que responda às necessidades das delicadas fases de transição, principalmente da adolescência à vida adulta, colocando o sujeito no centro do projeto, podendo decidir sobre as melhorias de suas condições de vida e suas possibilidades de inclusão. Acreditamos que as trajetórias de vida devam ter sentido para as pessoas com deficiência e isso não poderá ser realizado se a rede de serviços sócio-sanitários pararem de apoiar os sujeitos e suas famílias, em fases delicadas de transição (GIACONI, 2012b). O prolongamento da vida em contexto familiar, com a utilização de ambientes assistivos personalizados, pode ser uma ótima resposta.

\subsection{Algumas Experiências I nternacionais}

O panorama internacional é rico de experiências. A seguir, ilustramos alguns projetos europeus seguindo o critério cronológico na exposição, como forma de evidenciar os passos evolutivos da pesquisa em Ambient Assisted Living.

\subsubsection{Projeto BESTA na Noruega}

Em 1995 foram financiadas experiências para o planejamento, implementação e execução de sistemas domésticos inteligentes para apoiar pessoas mais necessitadas. O projeto de pesquisa, denominado BESTA, permitiu realizar apartamentos domóticos, na cidade de Tonsberg, principalmente para pessoas com diversos tipos de demência. As oito casas piloto foram construídas após numerosas solicitações, por parte de famílias de pessoas com demência. Os motivos das solicitações eram principalmente ligados à ansiedade constante com relação aos parentes com alto risco de quedas, incêndios e fugas. 
As instituições locais se organizaram, mediante a adoção do sistema ElB4, conseguindo dar vida ao Projeto Besta, que visava pesquisar modalidades adequadas para transformar as casas em locais mais seguros e facilitar a cotidianidade das famílias e o trabalho de assistentes. Buscava também demonstrar que habitações domóticas podem oferecer uma maior qualidade de vida para pessoas com deficiência, em comparação com clínicas de internação.

As diversas soluções foram escolhidas individualmente pelos residentes, em cooperação com os familiares e pessoal de assistência. Os dispositivos aplicados nas oito casas foram: sensor no ingresso principal, sensores nas portas que iam do apartamento ao jardim, monitor no fogão, sensor na cama a partir do peso, detectores de fumaça e aquecimento elétrico no piso.

Um elemento a destacar foi o envolvimento ativo dos cuidadores/assistentes e dos familiares, na seleção de dispositivos e na aprendizagem sobre modalidades de gestão do sistema domótico, fornecidas pelos técnicos. Isso demonstra a consciência sobre os efeitos positivos da combinação entre cuidados e tecnologias, tanto que os envolvidos declaravam perceber o sistema como um elemento suplementar à segurança no trabalho.

\subsubsection{Projeto Asura Flats na Espanha}

O complexo residencial realizado em Madridé apresentado como um modelo inovador para a QV e é destinado a pessoas com deficiência. É composto de quatro edifícios independentes, interligados pelo andar térreo, onde se encontram todos os serviços, como sala de jantar, lanchonete, escritórios, etc. Pode hospedar até 43 pessoas e é dotado de serviços acessíveis, oferecendo o máximo de inovações tecnológicas em todos os espaços.

O projeto Asura flats recebeu o nome da localidade na qual foi construído, uma zona central de Madrid. A localização facilita o acesso a todos os tipos de serviço, porque é atendido por transporte público. A principal característica inovadora dessa construção é que as pessoas que vivem nos apartamentos podem projetá-los, decorá-los e personalizá-los com base em gostos pessoais e em suas necessidades, usufruindo de serviços atentos aos cuidados individuais. Cada apartamento é completamente automatizado e consente à pessoa com deficiência independência em sua vida cotidiana. São especialmente enfatizadas a decoração, o design e a organização dos espaços para que as pessoas se sintam em casa. Para que isso seja possível, o ambiente deve ser prazeroso e confortável.

A estrutura do complexo residencial consegue conjugar dois aspectos: por um lado, a privacidade, mediante a pré-disposição de espaços de uso individual que consentem ter a máxima autonomia pessoal e a intimidade preservados; por outro, o compartilhamento de espaços comuns, como restaurantes, lanchonetes, lavanderia, enfermaria e espaços para atividades de lazer.

\footnotetext{
${ }^{4}$ European Installation Bus, oulnstabus. Sistema descentralizado para controlar dispositivos elétricos em uma residência.
} Desenvolvido para combinar economia energética, segurança, conforto e flexibilidade. 


\subsubsection{Projeto SmartBo na Suécia}

O projeto financiado pela Swedish Handicap Institute é destinado a pessoas com mobilidade reduzida, com déficits sensoriais ou com deficiência cognitiva (do desenvolvimento, lesões cerebrais, demência) e realizou, em 2010, um apartamento JK, térreo, em um edifício de cinco andares, situado no subúrbio de Estocolmo.

O projeto concentrou-se em dispositivos de tecnologias da informação e da comunicação (TIC) de assistência e soluções realizadas com tecnologia informática e eletrônica. O objetivo é demonstrar como pessoas com deficiência podem viver uma vida autônoma e rica, independente do tipo de deficiência. Para isso, as funções do apartamento podem ser controladas e supervisionadas, de modo a garantir segurança para pessoas com leve ou grave deficiência. Além disso, A SmartBoé uma casa com estrutura para relax e lazer, para trabalho e estudo.

Na seleção de instalações mais funcionais, para satisfazer as exigências dos moradores, optaram por equipamentos que podem ser subdivididas em duas categorias, comum a todos e adaptada a cada grupo, incluindo também soluções individuais. A primeira categoria compreende os sistemas de base, que consentem controlar funções da casa, como janelas, portas, fechaduras, torneiras, energia elétrica e fogão. A segunda categoria é constituída de dispositivos que permitem a supervisão e controle das pessoas por meio de interfaces adaptadas a cada uma delas. Esse é o verdadeiro desafio em um projeto como o SmartBo, que busca otimizar a acessibilidade para pessoas com vasta gama de deficiências.

A interface gráfica do programa, idealizado pelo SmartBo, permite a visualização do alto de todo o apartamento. A pessoa clica para entrar em uma peça, ou utiliza a letra inicial do nome da peça ( q para quarto, etc.) e controlar os objetos. Por exemplo, pode-se ver se uma lâmpada está acessa, se uma janela ficou aberta, etc. é possível trocar a lâmpada ou desativá-la clicando no mouse, ou em uma letra no teclado, ou ainda, tocando o pictograma apropriado no teclado alternativo. Pode-se abrir ou fechar a janela da cozinha da mesma forma. Para cada objeto controlável existe uma série de níveis de alerta a escolher.

Para os objetos mais importantes a serem verificados frente a qualquer mudança, os painéis são visualizados na tela do computador, às vezes com um anúncio vocal. Por exemplo, o alarme é ativado e começa a tocar a campainha ou o telefone, pode ser porque o fluxo d'água de uma torneira aumentou, o fogão permaneceu acesso ou muito quente, etc. Para pessoas com deficiência auditiva e visual, alguns avisos são transmitidos a um pequeno dispositivo vibratório, a ser usado na cintura, ou no bolso. São diversos tipos de vibração, dependendo do tipo de aviso, por exemplo, campainha, Test-Driven Development (TDD), Braille-telefone, alarme anti-incêndio, etc.

Todos os computadores em SmartBo são coligados entre si e em uma rede wireless, assim pode-se movimentar livremente, por exemplo com a cadeira de rodas, e controlar o apartamento à distância. Ao lado da cama e da porta de entrada ficam posicionadas duas caixas, Boa noite e Até logo, com um botão pulsante, um LED vermelho e outro verde. Apertando o botão quando sai do imóvel, ou de noite quando vai dormir, obtem-se uma 
mensagem de Tudo bem, ou uma mensagem que avisa o que foi esquecido de ser feito. As mensagens são orais, visuais (vermelho e verde nas caixas) ou táteis (barras salientes na parte inferior da caixa). Desse modo, as informações podem ser recebidas independente da deficiência.

Dentro da casa, por meio do computador, existem vários outros serviços disponíveis. Esses incluem uma impressora colorida, um scanner e um programa Optical Character Recognition (OCR), para que as pessoas que não veem ou não escutam possam scannear um texto para visualização em Braille, e um software do modem para o acesso a internet.

\subsection{Algumas Experiências na I tália}

A seguir, apresentamos projetos realizados na Itália, nos últimos anos ${ }^{5}$. Todos derivam da constatação de que o binômio domótica e deficiência devam ser estudados juntos, mas cada projeto diferencia-se dos outros pelo tipo de abordagem, realização e deficiências enfatizadas. De novo, o critério cronológico é seguido pelas razões acima colocadas: evidenciar os passos evolutivos da pesquisa multidisciplinar nessa área. O último projeto apresentado vai além da temática da deficiência, porque diz respeito à relação entre domótica e envelhecimento ativo/longevidade, mas é parte da discussão deste artigo porque se insere na visão global de Ambient Assisted Living.

\subsubsection{Projeto em Direção à Autonomia: da eficiência às habilidades diversas ${ }^{6}$}

Em 2010 foi inaugurada a casa domótica para pessoas com deficiência em Beregazzo, na Província de Como. O projeto foi realizado pela cooperativa social Sim-patia, que escolheu a domótica para desenvolver uma residência para pessoas com deficiências graves como tradução de melhorias tangíveis, em termos de condições de vida e autonomia na gestão dos espaços e instrumentos da casa. Os destinatários eram pessoas com limitações físicas graves, com mobilidade reduzida dos membros inferiores e/ou superiores, mas com manutenção de discreta capacidade cognitiva.

Os primeiros três apartamentos foram dotados de equipamentos tecnológicos e sistemas domóticos de controle, com vistas a melhorar sensivelmente as condições de vida dos condôminos. Foram utilizados dispositivos touchscreen e outros sistemas automatizados, instalados diretamente em cadeiras de roda, para consentir o comando de diversas funções: abertura e fechamento de persianas, janelas e portas, acesso às funções da cozinha, da televisão e do computador.

O cotidiano das pessoas com deficiência é monitorado por educadores sociais, regularmente em contato com os moradores dos apartamentos, e para a gestão dos cuidados

\footnotetext{
5 Por questões de espaço, optamos por não incluir algumas importantes experiências: o projeto Domótica: Auxílios e Terapia Ocupacional (DAT), em Milão, realizado pela Fondazione Don Carlo GnocchiOnlus que pode ser consultado em Andrich R., Gower V., Converti R.M., The DAT Service, anintegrated approach to improve independenceat home, Challenges for Assistive Technology, Ios Press, Amsterdam, p. 579-583; Projeto Distant-speech interaction for Robust Home Applications(DIRHA), coordenado pela Fondazione Bruno Kessler (Fbk) di Trento, em 2012; Projeto Domótica e Ambientes Formativos, realizado na Universidade de Florença, Região Toscana, em 2013, que visava realizar dispositivos que permitissem a inclusão escolar de crianças e adolescentes com deficiências motoras.

6 O projeto pode ser consultado em: <http://www. sim-patia. it/it/centro-ricerche>.
} 
higiênicos e assistenciais eles contam com operadores sócio-sanitários, duas vezes ao dia, manhã e tarde. Para garantir o necessário controle e segurança, foi prevista a possibilidade, somente se a pessoa desejar, de manter contato com uma central operativa diretamente na cooperativa Sim-patia. Uma vez recebido o telefonema, um operador da central pode visualizar a situação por meio de tele câmeras colocadas no apartamento, dotadas de um canal áudio para a comunicação. As pessoas com deficiência que desejam sair de casa, servindo-se de cadeira de rodas elétrica, dispõem de um dispositivo GPS, que permite enviar um alarme à central operativa indicando sua localização.

\subsubsection{Projeto Re-Freedom ${ }^{7}$}

O projeto Rede Funcional Para Pesquisa e Experimentação de Serviços Inovadores Domiciliares (Re-Freedom), promovido pela Prefeitura de Udine, Snaider oScientific Foundation e Friuli Innovazione e co-financiado pela Região Autônoma Friuli-Veneza-Giulia, foi iniciado em março de 2011. Seu objetivo era desenvolver um protótipo de rede de habitações na cidade de Udine, munida de tecnologias domóticas e TIC, de modo a experimentar formas inovadoras de serviços sócio-sanitários para pessoas com deficiência e idosos.

O projeto de pesquisa se referia a um núcleo mínimo de duas residências colocadas à disposição da prefeitura, adequadamente reestruturadas, onde as pessoas com deficiência pudessem experimentar diretamente o uso de tecnologias para melhorar a QV. Essas duas casas foram transformadas em unidades habitacionais-teste. Destinado principalmente a pessoas com dificuldade de locomoção, as casas foram equipadas com tecnologias domóticas, facilitando a mobilidade e a acessibilidade. Mais especificamente, foram instalados sensores ambientais (para alagamento, fuga de gás e fumaça) que, além de assinalar o perigo por meio de alarmes sonoros e luminosos, foram ligadas as eletroválvulas que se fecham automaticamente em situações de risco. Sensores de movimento, comando das luzes, automação do portão de entrada, automação da porta interna e zona de serviços, foram todos ligados ao sistema domótico, podendo ser controlados automaticamente pela interface, ou integrados em séries de comandos automáticos ligados entre si. A acessibilidade física à zona de serviços foi garantida pela automação de portas de correr. O sensor de presença colocado no banheiro, que permite a atuação de luzes e portas, foi conectado a um marcador de passos que se acende quando o local está ocupado. A janela do banheiro também é monitorada e dotada de sensores aberto/fechado que, ligados a uma central de alarmes, mandam sinais ao sistema de abertura anômala, para garantir a segurança contra invasões, ou simplesmente manda a informação de que a janela está aberta, com a possibilidade de fechá-la sem necessidade de deslocamento físico. Para a segurança do uso do banheiro, uma corda ao lado do chuveiro foi ligada ao sistema de alarme. Em caso de perigo, é possível desbloquear a porta pelo lado de fora, manual ou automaticamente, por meio do sistema domótico.

O segundo apartamento-teste foi destinado a usuários com deficiências sensoriais e cognitivas, tendo como critério fundamental de escolha do mobiliário a fluidez possível de

\footnotetext{
${ }^{7}$ O projeto pode ser consultado em: <http://www.sim-patia.it/it/centro-ricerche>.
} 
pessoas com esse tipo de déficit. $\mathrm{O}$ apartamento foi subdividido em três áreas funcionais: a sala de pesquisa, dotada de móveis normais de escritório, porque hospedava a maior parte das atividades de pesquisa e desenvolvimento de soluções assistivas; o ambiente cozinha equipado com acessórios para deficientes visuais; as outras peças foram mobiliadas com móveis de fácil reconhecimento e com minimização de risco, para que as pessoas pudessem conviver em situações típicas de uma vida cotidiana. Por exemplo, ângulos dos móveis arredondados, caracterização cromática de casa peça, uso específico de cores para porções de móveis e acessórios, alto contraste cromático entre portas e puxadores dos móveis.

\subsubsection{Projeto Fim de Semana de Autonomia em Apartamentos Domóticos}

O projeto foi apresentado em junho de 2012, com dois apartamentos, com 80 e 50 metros quadrados, na Área Auxílios de Corte Roncati, em Bolonha ${ }^{8}$. Os apartamentos foram inicialmente idealizados como laboratórios experimentais de soluções habitacionais domóticas de alta acessibilidade para, posteriormente, tornarem-se lugares de autonomia de vida cotidiana e veículos de melhora dos serviços oferecidos a pessoas com deficiência Assisted Technology Learning Through A Unified Curriculum (ATLEC), 2013.

Um único telecomando, ativável com um movimento da cabeça, pode gerir toda a casa, desde as luzes até o aquecimento, a cozinha, os banheiros, portas e janelas, sistemas de comunicação e entretenimento, cama elétrica, sistema de levantamento de pessoas por meio de movimento dos olhos, de comunicação, navegação internet, e muitos outros a partir de computador dedicado.

Durante os primeiros finais de semana vividos nos apartamentos, os jovens desenvolveram experiências significativas de autonomia pessoal, descobrindo novas possibilidades e testando ambientes favoráveis, com soluções técnicas assistivas. Utilizando os equipamentos durante as experiências, os jovens forneceram sugestões importantes aos técnicos para aprimoramento dos ambientes e soluções. Por exemplo, sugestões para melhorara utilização do chuveiro, solicitação de um dispositivo auxiliar para cozinhar, evitando ter que misturar a comida, para que a mesma não grude na panela, etc. A experimentação ainda está em curso, durante os fins de semana, como um verdadeiro living lab, na expectativa de que gere ainda muitos bons resultados.

\subsubsection{Projeto Accessible Light}

Foi apresentado em 2013 na Província de Ancona e é muito inovador em soluções assistivas para pessoas com deficiência auditiva. Accessible Lighté um sistema que utiliza tecnologias domóticas para eliminar barreiras de comunicação e incrementar segurança para pessoas surdas. É caracterizado pela ilumino técnica, que traduz o mundo visível, ou seja, transforma os efeitos sonoros em estímulos visuais, facilitando a acessibilidade de pessoas com deficiência auditiva.

\footnotetext{
${ }^{8}$ Disponível em: <http://www. ausilioteca. org/>.
} 
O sistema funciona por meio de um painel, com botões coloridos: os brancos operam o interfone, a campainha de ingresso e o despertador; os azuis piscam em casos de alagamento; os vermelhos, fuga de gás e incêndio; os verdes são os codificadores antifurto. O projeto foi idealizado sob uma ótica ampla para proporcionar a autonomia na vida cotidiana e vem sendo utilizado em lugares públicos, como hospitais, museus, entre outros.

\subsubsection{Projeto Longevity Hub}

O Centro Longevity Hub, a casa inteligente para longevidade ativa e independente do idoso por meio de tecnologias domóticas, foi inaugurado em 2014 na cidade de Fabriano, Província de Ancona. Foi desenvolvido pela colaboração entre a Região Marcas, o Ministério da saúde e Instituto Nacional de Pesquisa e Cuidado de Idosos (INRCA) e Itália Longeva. O projeto busca o desenvolvimento de modelos assistenciais alternativos e inovadores, como apoio ao envelhecimento. A tecnologia é pensada em duas grandes frentes: a consideração de que o idoso de hoje faz uso de tecnologia mais facilmente, e de que a mesma pode favorecer a assistência ao idoso e sua família. As tecnologias utilizadas vão da domótica ao Ambient Assisted Living, passando por dispositivos SmartObject.

O Centro desenvolverá ainda vários projetos, múltiplas funções, constituídos por três subsistemas: 1) o Sistema Laboratório (LAB) estruturado em LAB1, fases de estudo, pesquisa e desenvolvimento; LAB2, fases aplicativas, definição de protótipos para a casa inteligente; LAB3, implantação e experimentação das residências (HOME), subdividido em HOMEp, protótipo da casa e HOMEr a residência experimental. Além disso, o sistema NET (Network) terá a função de troca e divulgação de resultados obtidos.

Vários projetos se inserem no guarda-chuva Longevity Hub, direcionados às relações entre longevidade ativa e domótica: Projeto AALisabeth, de objetos inteligentes para monitorar a alimentação $^{9}$; Projeto HicMO, plataforma de telemedicina ${ }^{10}$, integrando eletrocardiógrafo, balança, medidor de pressão e de frequência cardíaca com outros objetos inteligentes da rotina cotidiana da residência; Projeto Hdomo 2.0, estudo dos comportamentos dos idosos, monitoramento de hábitos, para a prevenção de acidentes por meio de sistemas de visão automática e reconhecimento ${ }^{11}$; Projeto Pass, modelo de residência domótica para ser inserido em novas habitações quanto em outras já existentes ${ }^{12}$; Projeto Transparente ${ }^{13}$; Projeto II

\footnotetext{
9 Entidades envolvidas: MeTeDaS.r.I. (San Benedetto delTronto), MAC srl (Recanati) Universitàdeglistudidi Parma; UniversitàdeglistudidiCamerino; I.T.C. Srl (Recanati); SassomeccanicaSrl (San Benedetto delTronto); Coop. Soc. Nuova Ricerca Agenzia Res (MaglianodiTenna); Valdichienti (Tolentino); RalòSrl (San Severino Marche); ERS Elettronica (Ancona); On-Off Srl (San Benedetto delTronto); DienpiSrl (San Benedetto delTronto); EusebiSrl (Ripatransone).

10 Entidades envolvidas: Logical System Srl (Jesi) LaboratoriodelleldeeSrl (Fabriano); Elite SocietàdiElettronica per I'I nnovazioneTecnologica-Società Cooperativa (Fabriano); SpesSocietà Cooperativa per Azioni (Fabriano); Panatta Sport Srl (Apiro); InoxaSrl (Polverigi); EurosuoleSpa (Civitanova Marche); GreenergSrl (Pesaro); Maiolati Ivana (Macerata); DesiCucine (Cerreto D'Esi); Ondemand Group Srl (Chiaravalle); IndesitCompanySpa (Fabriano); BaxSrl (Pesaro); UniversitàPolitecnicadelle Marche (Ancona).

11 Entidades envolvidas: APRA Progetti S.R.L. (Jesi) R.I.Co. S.R.L. (Castelfidardo); Detron S.R.L. ( Civitanova Marche); Jef S.R.L. (Montegranaro); Eurosystems S.R.L. (Fermo); 4d Engineering S.R.L. ( Civitanova Marche); VidexElectronics S.P.A. (Monte Giberto); Univ. PolitecnicaDelle Marche; Tecnomarche S.C.A R.L. (Ascoli) ; Proietti Planet S.R.L. (Ascoli); Speech Village S.R.L. (Ascoli); Informa sistemi S.P.A. (Ancona); Safeway S.R.L.; (Ascoli); Ssg S.R.L. (Fabriano); Websolute S.R.L. (Pesaro); Genera S.C. Ar.L. (Ascoli); ConsorzioConier (Ancona); Tmltalia (Folignano).

12 Entidades envolvidas: IstitutodiRiabilitazione S. Stefano Srl (Porto PotenzaPicena), AditechSrI (Ancona); Team ItaliaSrI (Fano); FilippettiS.p.A.(Falconara); GPL Costruzioni Generali Srl. (Ancona); NautesS.p.A. (Jesi); SailmakerInternationalS.p.A (Ancona); Siam S.r.I. (Montemarciano); Sigma S.p.A (Altidona); Smart Space SolutionsS.r.I. (Ancona); Universitàdegli Studi diCamerino; H\&H Group S.r.I. (Serrungarina); Domingo SalottiS.r.I. (Pesaro).
} 
Marchingegno e laSibilla, sistema baseado em uma lâmpada LED, que integra um distribuidor de medicamentos e um sistema de monitoramento de deambulação ${ }^{14}$.

\section{Projeto e Personalização para uma Rede Inclusiva}

O cenário da QV e do Ambient Assisted Living promove reflexões pedagógicas, com vistas à construção de projetos para melhorias nas condições de vida das pessoas com deficiência. Na literatura científica internacional, Buntinx e Schalock (2010) propõem um quadro orgânico de avaliação que leva em consideração modelos de funcionamento humano, QV e criação de apoios. Nessa perspectiva, o aspecto relevante diz respeito à mudança de foco da avaliação de déficits e das deficiências, para apoios necessários às pessoas para que conduzam uma vida independente e para implementar a QV ${ }^{15}$.

Em outras palavras, a avaliação multidisciplinar orienta-se pelo conhecimento da pessoa do ponto de vista das habilidades intelectuais, do comportamento adaptativo, da participação, das interações e papéis sociais, da saúde e do contexto. Ao mesmo tempo considera as necessidades do sujeito e as áreas de intervenção, ou seja, os apoios necessários para a QV das pessoas com deficiência. Tudo isso para projetar programas direcionados, empenhar recursos e predispor apoios voltados à melhoria das condições de vida.

Um primeiro aspecto a considerar diz respeito à prática profissional de projeto de ambientes assistivos, que deve ser realizada de forma interdisciplinar. Existem muitos elementos a serem levados em conta em projetos dessa natureza, além da inclusão e da personalização dos recursos. Em termos amplos, considerando as fases evolutivas da pessoa com deficiência, existe o risco da fragmentação das intervenções educativas nos diversos momentos de vida e, como já referido, o risco de recorrer a soluções emergenciais sem levar em conta esse histórico. Isso é o que se costuma chamar de intervenções de eterno presente, nas quais os objetivos educacionais aparecem e desaparecem nos diversos anos de vida. Nos últimos anos, vários são os estudos que defendem a necessidade de uma perspectiva life-span, ou seja, de um projeto de investimento ao longo da existência. É o reconhecimento da necessária continuidade das intervenções educativas, por meio de percursos eficazes visando a promoção de melhores condições de vida e o desenvolvimento da QV.

Um segundo aspecto, diz respeito à simplificação linear da problemática, do diagnóstico funcional e do perfil dinâmico, do sujeito com deficiência, sobre a qual vem construído o projeto de intervenção. Isso resulta em um projeto desalinhado com as reais necessidades da pessoa, uma simples adaptação à estrutura, sem perspectiva inclusiva.

Um terceiro aspecto, quando a pessoa com deficiência perde a família os projetos de Ambient Assisted Living não parecem factíveis e, muitas vezes, a opção passa a ser a internação em uma grande estrutura sanitária. Gostaríamos de evidenciar a possibilidade de alinhar o projeto de vida de uma pessoa com deficiência com o paradigma da QV, integrando a

${ }^{13}$ Entidades envolvidas: AtlcSrl (Ancona), UniversitàPolitecnicadelle Marche; UniversitàdiCamerino; AutomaSrl (Monsano); SalvatelliSrl (Montegranaro); RS Elettronica (Osimo); INIM Srl (Ancona); ArieLABSrl (Ancona); Iselqui Technology SrI (Ancona); DUEBBI Snc (Civitanova Marche); IDEA Scarl (Ancona); Tre P EngineeringSrl (Chiaravalle).

14 Entidade Envolvida: Semar (Ancona).

15 É utilizada a Escala de Intensidade de Apoio (SIS), adaptada para a realidade italiana (COTTINI et al., 2008). 
pessoa com o seu contexto de referência. A literatura científica concentra-se sobre o conceito de alinhamento (BIGGS, 2003, LAURILLARD, 2012; ROSSI, 2011), conceito central em projetos educativos e de vida. Muitas são as dimensões a serem alinhadas em um projeto educativo e dependem da ação de bons profissionais, copartícipes na construção de alternativas, incluindo ambientes de vida assistivos.

Existem várias formas de alinhamento (GIACONI; RODRIGUES, 2014). Uma forma de alinhamento parte das condições de funcionamento da pessoa, obtidas pela avaliação multidisciplinar, levando em conta os domínios da QV. A avaliação permite conhecer as condições da pessoa, seus pontos fortes e limitações. Outra forma seria o alinhamento dos objetivos das intervenções necessárias, a fim de construir junto à pessoa o melhor caminho para sua inclusão e o reconhecimento de seu estatuto identitário de adulto. O projeto, portanto, deve partir das necessidades de apoio, que correspondem às áreas nas quais a pessoa não atinge uma adequada e satisfatória QV.

Nessa ótica, ocorre um processo de individuação e priorização de intervenções e mapeamento de recursos, apoios indispensáveis e possíveis. É importante correlacionar as questões educativas com os aspectos contextuais e pessoais. Se essa correlação não acontece observa-se uma grande discrepância entre as condições funcionais e os níveis da QV (SCHALOCK et al., 2007). Entra em jogo um outro tipo de alinhamento, ou seja, entre desejos, expectativas e necessidades da pessoa com deficiência, de seus familiares e os dos profissionais envolvidos. Subestimar essa necessidade de alinhamento, em uma visão sistêmica e ecológica, de envolvimento de todos no projeto de vida da pessoa com deficiência, pode comprometer a realização de um sistema integrado e funcional de QV.

Os objetivos das diversas intervenções e profissionais envolvidos no projeto de vida (clínico, reabilitativo, educativo e social) precisam ser orientados à promoção das condições de vida (COTTINI et al., 2008). Os objetivos devem ser escolhidos visando o desenvolvimento de competências, de autonomia adulta, ou seja, competências ligadas a autodeterminação, a manutenção de rede de apoio, a gestão consciente da própria saúde, do lazer, da ocupação, da identidade e auto-projeção (IANES; CRAMEROTTI, 2009). Além disso, devem ser relevantes e significativos no projeto de vida pessoal, favorecendo a realização de necessidades cotidianas, a participação social em redes de familiares, amigos e comunidade (ADAMS et al., 2006).

Wehmeyer e colaboradores demonstraram que a autodeterminação ${ }^{16}$ prescinde de condições ambientais disponíveis (must) e de condições de funcionamento. Pesquisas evidenciaram como a autodeterminação é uma dimensão crucial para melhorar a QV e para favorecer a inclusão social (LACHAPELLE et al., 2005). Um alto nível de autodeterminação aumenta a possibilidade de ter uma vida adulta positiva, por exemplo, no trabalho, com autonomia e independência ${ }^{17}$.

\footnotetext{
${ }^{16}$ Pesquisas internacionais promoveram procedimentos de avaliação de autodeterminação para pessoas com deficiência, seja por meio de instrumentos de auto-avaliação, que de hetero-avaliação (GIACONI, 2015).

17 O nível de satisfação com a vida é muito ligado ao nível de autodeterminação. Nas pessoas com deficiência isso é ainda mais marcado.
} 
Para a avaliação de projetos educativos podemos formular algumas questões norteadoras, para profissionais que querem pensar à promoção de ambientes de vida assistivos. Nesse propósito, apresentamos no Quadro 2 um esquema, adaptado a partir de diversos estudos (ADAMS et al., 2006).

Quadro 2 - Esquema de Avaliação de Projetos Educativos.

\begin{tabular}{|c|c|}
\hline Indicadores & Descritores \\
\hline Relevância & $\begin{array}{l}\text { Os objetivos reabilitativos são } \\
\text { relevantes e significativos no projeto } \\
\text { de vida individual? }\end{array}$ \\
\hline Observabilidade & $\begin{array}{l}\text { Os objetivos são traduzíveis em } \\
\text { comportamentos observáveis? }\end{array}$ \\
\hline Adequação & $\begin{array}{l}\text { São apropriados à idade cronológica do } \\
\text { sujeito e às condições de saúde? }\end{array}$ \\
\hline Funcionalidade & $\begin{array}{l}\text { São funcionais às necessidades de vida } \\
\text { cotidiana e significativos com relação } \\
\text { aos contextos de pertencimento? }\end{array}$ \\
\hline Temporalidade & $\begin{array}{l}\text { São temporalmente ponderados e } \\
\text { limitados no tempo para verificar o seu } \\
\text { atingimento? }\end{array}$ \\
\hline Monitoramento & $\begin{array}{l}\text { São monitoráveis e monitorados em } \\
\text { termos de melhoras ou de eventuais } \\
\text { pioras? }\end{array}$ \\
\hline Mensurabilidade & São qualificáveis e quantificáveis? \\
\hline Realismo & $\begin{array}{l}\text { São atingíveis em relação a recursos } \\
\text { humanos e materiais disponíveis? }\end{array}$ \\
\hline I nclusão & $\begin{array}{l}\text { São funcionais para acrescentar } \\
\text { autonomia, autodeterminação, } \\
\text { empowerment da pessoa, são } \\
\text { alinhados aos seus desejos? }\end{array}$ \\
\hline Participação & $\begin{array}{l}\text { Favorecem a participação social e as } \\
\text { relações de rede de amizade, família e } \\
\text { social? }\end{array}$ \\
\hline
\end{tabular}

Fonte: Giaconi (2015).

\section{Conclusões e Perspectivas}

A complexidade do cuidado de adultos com deficiência coloca alguns desafios à pedagogia especial. A primeira é seguramente a de repensar um plano estratégico capaz de apoiar e garantir projetos de vida correspondentes às necessidades das pessoas e orientados a atingir as melhores condições possíveis de vida.

A reflexão, mesmo se ligada à fase adulta, não pode deixar de levar em conta as diversas fases evolutivas e as emergências pedagógicas ao longo da vida. Ao mesmo tempo, não pode deixar de caracterizar-se como uma ação projetiva coparticipada e compartilhada: a pessoa com deficiência e sua família devem estar no centro de uma rede de sistemas integrados, direcionados à plena realização do projeto de vida.

Nessa corresponsabilidade e visando novos modelos de inclusão, como os ambientes assistivos discutidos nesse artigo, as palavras chaves devem ser: projeto, continuidade e formação. O projeto permite olhar para o futuro, em um jogo de alinhamento entre passado, presente e futuro, intencionalmente orientado à plena realização da identidade adulta e de uma vida de qualidade. Somente com essa perspectiva pode-se pensar em dar um real e concreto sentido na trajetória de vida da pessoa com deficiência e de sua família. 
A continuidade garante uma intencional sinergia de intervenções educativas que, certamente, mudam com o tempo e com relação às necessidades da idade do sujeito. Essas intervenções antecipam e apoiam as diversas fases de transição, em um tempo e espaço do que já foi realizado e o que pode ainda ser feito para a construção da identidade da pessoa com deficiência.

A formação, apoiada por dispositivos adequados e práticas educativas finalizados à reflexão sobre posturas e representações de profissionais, torna-se a estrada para garantir um processo real de profissionalização e de mudança no cuidado de adultos com deficiência e de suas famílias.

\section{Referências}

ADAMS, L. et al. Individual Planning: an exploration of the link between quality of plan and quality of life. British Journal of Learning Disabilities, West Sussex, n. 34, p. 68-76, 2006.

BIGGS J. B. Teaching for Quality Learning at University. Buckingham: Open University, 2003.

BORTHWICK-DUFFY, A. Quality of Life and Quality of Care in Mental Retardation. In: ROWITZ (Ed.). Mental Retardation in the Year 2000. New York: Springer, 1992. P. 52-66.

BROWN, I.; BROWN, R. I. Choice as an Aspect of Quality of Life for People with Intellectual Disabilities. J ournal of Policy and Practice in Intellectual Disabilities, Malden, n. 6, p. 11-18, 2009.

BROWN, R. I. Aging, Disability and Quality of Life: a challenge for society. Canadian Psychology, Winnipeg, CA, v. 30, n. 3, p. 551-559, 1989.

BROWN, R. I. (Ed.). Quality of Life for Handicapped People. London: Croom Helm, 1988.

BUNTINX, W. H. E.; SCHALOCK, R. L. Models of Disability, Quality of Life and Individualized Supports: implications for professional practices. J ournal of Policy and Practice in Intellectual Disabilities, Malen, v. 7, n. 4, p. 283-294, 2010.

CASAZZA, S. et al. Anziani: trabisogni in evoluzione e risposte innovative. Milano: Franco Angeli, 2002.

CAMBRIDGE, P.; CARNABY, S. Making it Personal: providing intimate and personal care for people with learning disabilities. Brighton: Pavilion, 2000.

CAMPO, S. F. et al. Measurement Characteristics of the Quality of Life Index when Used with Adults Who Have Severe Mental Retardation. American J ournal on Mental Retardation, Washington, US, n. 100, p. 546550, Mar. 1996.

COTTINI, L.; FEDELI, D. Invecchiamento e Qualitàdella Vita: La QV come quadro concettuale per l'analisi e per l'intervento nel decadimento cognitivo dele persone conritardo mentale. American Journal on Mental Retardation, Washington, US, v. 5, n. 3, p. 375-382, 2007.

COTTINI, L. et al. La Supports Intensity Scalenel Panorama Riabilitativo Italiano: standar dizzazione e procedure psicometriche. American Journal on Mental Retardation, Washington, US, v. 6, n. 1, p. 21-38, 2008.

DENNIS, R. et al. Quality of Life as Context for Planning and Evaluation of Services for People with Disabilities. Exceptional Children, Reston, Va., n. 59, p. 499-512, 1993.

EMERSON, E. et al. The International Handbook of Applied Research in Intellectual Disabilities. Chichester: Wiley, 2004. 
FELCE, D.; PERRY, J. Exploring Current Conceptions of Quality of Life. In: RENWICK, I.; BROWN, I.; NAGLER, M. (Ed.). Quality of Life in Health Promotion and Rehabilitation: conceptual approaches, issues, and applications. Thousand Oaks: Sage, 1996. P. 51-62.

FELCE, D. et al. The Effectiveness of Staff Support: evaluating active support training using conditional probability approach. Research in Developmental Disabilities, Atlanta, GA, v. 21, n. 4, p. 243-255, 2000.

FLANAGAN, J.C. A Research Approach to Improving our Quality of LIfe. American Psychologist, Washington, US, n. 33, p. 138-147, 1978.

GIACONI, C. Nella Comunitàdi Capodarcodi Fermo: dalle praticheal I'assetto pedagógico condiviso. Roma: Armando, 2012a.

GIACONI, C. Disability, Citizenship and Democracy from Life Plan to Social Inclusion. Civitas Educationis: education, politics and culture, Napoli, v. 1, n. 2, p. 91-104, 2012b.

GIACONI, C.; RODRIGUES, M. B. Organização do Espaço e do Tempo na Inclusão de Sujeitos com Autismo. Educação \& Realidade, Porto Alegre, v. 39, n. 3, p. 687-705, 2014.

GIACONI, C. Qualità dela Vita e Adulti con Disabilità. Milano: Franco Angeli, 2015.

IANES, D.; CRAMEROTTI, S. II Piano Educativo Individualizzato, Progetto di Vita: raccolta di materiali, strumenti e attività didattiche. 9. ed. Trento Erickson, 2009. V. 2.

LACHAPELLE, Y. et al. The Relationship Between Quality of Life and Self-determination: an international study. Journal of Intellectual Disability Research, Oxford, n. 49, p. 740-744, 2005.

LANDESMAN, S. Quality of Life and Personal Life Satisfaction: definition and measurement issues. Mental Retardation, Washington, US, n. 24, p. 141-143, 1986.

LAURILLARD, D. Teaching as a Design Science. London: Routledge, 2012.

LIFSHITZ, H.; MERRICK, J.; MORAD, M. Health Status and ADL Functioning of Older Persons with Intellectual Disability: community residence versus residential care centers. Research in Developmental Disabilities, Atlanta, GA, n. 29, p. 301-315, 2008.

MAES, B. et al. Quality-enhancing Interventions for People with Profound Intellectual and Multiple Disabilities: a review of the empirical research literature. Journal of Intellectual and Developmental Disability, London, v. 32, n. 3, p. 163-78, 2007

PENNE, A. et al. Staff Interactive Style During Multi-sensory Storytelling with Persons with Profound Intellectual and Multiple Disabilities. Journal of Intellectual Disability Research, London, v. 56, n. 2, p. 167178, 2012.

PETRY, K.; MAES, B.; VLASKAMP, C. Psychometric Evaluation of a Questionnaire to Measure the Quality of Life of People with Profound Multiple Disabilities (QOL-PMD). Research in Developmental Disabilities, Atlanta, GA, n. 30, p. 1326-1336, 2009.

RASHIDI, P.; MIHAILIDIS, A. A Survey on Ambient-Assisted Living Tools for Older Adults. IEEE Journal of Biomedical and Health Informatics, New York, v. 17, n. 3, p. 579-590, 2013.

REITER, S.; SCHALOCK, R. L. Applying the Concept of Quality of Life to Israeli Special Education Programs: a national curriculum for enhanced autonomy in students with special needs. International Journal of Rehabilitation Research, London, n. 31, p. 13-21, 2008.

RENWICK, R.; BROWN, I. Being, Belonging, Becoming: the Centre for Health Promotion model of quality of life. In: RENWICK, R.; BROWN, I.; NAGLER, M. (Ed.). Quality of Life in Health Promotion and Rehabilitation: conceptual approaches, issues, and applications. Thousand Oaks, CA: Sage, 1996. P. 75-88.

ROSSI, P. G. Didattica e Nattiva. Milano: Franco Angeli, 2011. 
SCHALOCK, R. L. Three Decades of Quality of Life: focus on autism and other. Developmental Disabilities, Austin, v. 15, n. 2, p. 116-127, 2000.

SCHALOCK, R. L. et al. Conceptualization, Measurement, and Application of Quality of Life for Persons with Intellectual Disabilities: report of an international panel of experts. Mental Retardation, Washington, US, v. 40, n. 6, p. 457-470, 2002.

SCHALOCK, R. L.; VERDUGO ALONSO, M. A. Handbook of Quality of Life for Human Service Practitioners. Washington, DC: American Association on Mental Retardation, 2002.

SCHALOCK, R. L.; VERDUGO ALONSO, M. A. Manuale della Qualità della Vita: modelli e pratichedi intervento. Brescia: Vannini, 2006.

SCHALOCK, R. L.; GARDNER, J. F.; BRADLEY, V. J. Quality of Life for People with Intellectual and Other Developmental Disabilities: applications across individuals, communities, and systems. Washington, DC: American Association on Intellectual and Developmental Disabilities, 2007.

SCHALOCK, R. L. et al. Evidence-based Practices in the Field of Intellectual and Developmental Disabilities: an international consensus approach. Evaluation and Program Planning, New York, n. 34, p. 273-282, 2011.

THOMPSON, W. W. et al. Health-related Quality of Life Among Older Adults With and Without Functional Limitations. American Journal of Public Health, Washington, US, v. 102, n. 3, p. 496-502, 2012.

TIMMONS, V.; BROWN, R. I. Quality of Life Issues for Children With Handicaps. In: BROWN, R. I. (Ed.). Quality of Life for Handicapped People. 2. ed. Cheltenham: Stanley Thomas, 1997.

WEHMEYER, M. L. Self-determination and the Education of Students with Mental Retardation. Education and Training in Mental Retardation, Reston, Va, n. 27, p. 302-314, 1992.

ZIJ LSTRA, H. P.; VLASKAMP, C. Leisure Provision for Persons With Profound Intellectual and Multiple Disabilities: quality time or killing time? J ournal of Intellectual Disability Research, London, v. 49, n. 6, p. 434-448, 2005.

Recebido em 19 de julho de 2015

Aprovado para publicação em 21 de setembro de 2015

Catia Giaconi

Università degli Studi di Macerata, Macerata MC, Itália, catia.giaconi@unimc.it

Michela Carbonari

Università degli Studi di Macerata, Macerata MC, Itália, michela.carbonari@libero.it 